\section{COURRIER}

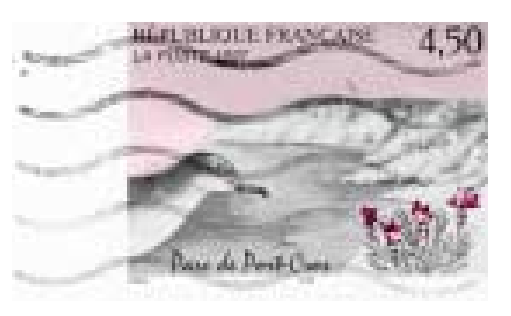

\title{
Pharmacie et génomique : réponse à Robert Vachy
}

Le marché américain du médicament est caractérisé par des prix de vente élevés des nouveaux produits, mais aussi par la forte place des produits génériques, c'est-à-dire de copies de médicaments originaux tombés dans le domaine public. Ainsi, il est habituel que les génériques prennent jusqu'à $80 \%$ du marché quelques mois à peine après l'expiration du brevet protégeant la molécule copiée: c'est arrivé récemment à l'anti-ulcéreux vedette, la cimétidine. Cela est dû, notamment, aux pressions très fortes des assurances privées sur les médecins et sur les malades. Les laboratoires pharmaceutiques américains tentent de réagir en fabriquant eux-mêmes le produit générique, ou en cassant les prix du produit original. En fait, la seule parade efficace est de rendre désuète la molécule passée dans le domaine public, en mettant au point un nouveau médicament plus performant. Cette recherche de l'innovation à tout prix, même s'il s'agit d'un progrès minimal, conduit à l'inflation des coûts d'une recherche souvent mal contrôlée, et au développement prioritaire de molécules dérivées du produit leader (ce qu'on appelle dans le jargon de la pharmacie des me too). Ces "pseudo-nouveaux » produits seront menés à terme à grand frais mais, en fait, sans grand risque et, avec un marketing aggressif, sauront grignoter quelques parts du marché de la nouvelle classe thérapeutique. Au total, si le coût total des sommes engagées par l'industrie mondiale du médicament est estimé à 400 milliards de dollars, seules 2 ou 3 molécules vraiment nouvelles sont mises sur le marché chaque année. Les médecins français qui lisent la revue indépendante Prescrire le savent d'ailleurs depuis de nombreuses années! L'efficience de la recherche industrielle en pharmacie est donc très faible (rendement réel de moins de $1 \%$ ).

Pourquoi investir dans la génomique ? Nous observons simplement que le phénomène des sociétés de biotechnologie spécialisées en génomique est très soutenu par les sociétés de «capital risque» et par le marché boursier américain qui, à certains égards, s'apparentent à une certaine loterie. Connaissant la puissance financière colossale des fonds de pension américains, investir un peu dans des sociétés «à risque» ne constitue pas un risque majeur, loin s'en faut. Pour les entrepreneurs, le risque de créer ces nouvelles activités est probablement beaucoup plus grand en France, pays où les banques, connues pour leur faible esprit d'aventure, leur réclament d'y investir leurs économies, voire d'y hypothéquer leurs biens. Quant aux retombées de la génomique, il est encore un peu tôt pour savoir si elles seront «juteuses» ou non. Nous espérons simplement qu'elles ne seront pas simplement un moyen d'enrichissement spéculatif, mais qu'elles contribueront significativement à aider l'industrie du médicament à améliorer la qualité de sa recherche fondamentale.

L'expérience américaine montre que les systèmes privés d'assurance maladie sont plus chers et moins équitables que les sytèmes universels de couverture sociale. Nous pensons que la santé ne doit pas être un bien de consommation comme un autre mais un droit inaliénable. La solidarité est le principe fondateur de la Sécurité sociale, et tout indique que notre peuple y est très attaché. Nous pensons que le temps des asiles pour indigents est révolu, et que chacun doit avoir droit à des soins de qualité. L'irresponsabilité mentale nous semble plutôt du côté de ceux qui pronent la destruction pure et simple de notre système de protection sociale. La résorption du «trou de la Sécurité sociale» passe certainement par une véritable politique de prévention et de dépistage précoce des maladies, par une meilleure formation des médecins et des malades; plutôt que par la suppression de l'assurance maladie, qui aggraverait les conditions sanitaires de l'ensemble de la population et, par là même, sa capacité de créer et de produire

\section{Philippe Froguel \\ Institut Pasteur de Lille, 1, rue Calmette, BP 245, 59019 Lille Cedex, France.}

